\title{
De Novo Sequencing of Peptides Using MALDI/TOF-TOF
}

\author{
Alfred L. Yergey, Jens R. Coorssen, Peter S. Backlund, Jr., Paul S. Blank, \\ Glen A. Humphrey, and Joshua Zimmerberg \\ Laboratory of Cellular and Molecular Biophysics, National Institute of Child Health and Human Development, \\ Bethesda, Maryland, USA
}

\author{
Jennifer M. Campbell and Marvin L. Vestal \\ Applied Biosystems, Framingham, MA, USA
}

\begin{abstract}
The recently developed MALDI TOF-TOF instrument yields relatively complex but interpretable fragmentation spectra. When coupled with a straightforward sequence extension algorithm, it is possible to develop complete peptide sequences de novo from the spectra. This approach has been applied to a set of peptides derived from typtic digestion of electrophoretically separated sea urchin egg membrane proteins. When directed to proteins that have been described previously, the results were in essential agreement with those obtained by conventional data base searching approaches, with certain important exceptions. The present method detected errors in published sequences and was able to develop sequences from peptides differing in mass by one dalton $(\mathrm{Da})$. These results show both the power of the present approach and the need for using de novo methods more frequently than may be otherwise appreciated. (J Am Soc Mass Spectrom 2002, 13, 784-791) @ 2002 American Society for Mass Spectrometry
\end{abstract}

$\mathrm{M}$ ass spectrometry has become an invaluable tool for the characterization of proteins, and can be used to measure the molecular weights of intact polypeptides determining peptide identity and delineate peptide structure. Identification of biochemically isolated proteins that are represented in data bases, either as translations of polynucleotide sequences or as independently determined amino acid sequences, may be the largest current application of mass spectrometry in the emerging field of proteomics. Protein identifications are accomplished using several approaches, often in combination. MALDI-TOF is the first method employed; exact masses of peptides resulting from endoprotease digestions are used in conjunction with a number of data base searching routines [1-7]. An alternative strategy is the use of MS/MS to obtain mass spectra of peptide fragment ions from a particular peptide precursor ion. These MS/MS approaches tend to be dominated by fragment spectra produced in multiple low energy collision reactions either in triple quadrupole or ion trap instruments. To identify the proteins, the observed low energy fragment ion spec-

Published online May 15, 2002

Address reprint requests to Dr. A. L. Yergey, Laboratory of Cellular and Molecular Biophysics, NIH, Building 10, Room 9D52, MSC 1580, Bethesda, MD 20892, USA. E-mail: aly@helix.nih.gov

"Also at: Neuroscience Research Group, Department of Physiology and Biophysics, University of Calgary, Alberta, CN. trum is matched with one that is predicted for a peptide of the same precursor mass found in a data base [8]. An alternative to spectra matching is the use of the sequence tag, a short string of sequence surrounded by two regions of known mass, developed by Mann and colleagues [9].

A fundamental assumption made by any of the various data base searching routines is that the proteins that were originally isolated for mass spectrometric study are described in a data base either specifically or in a closely homologous form. As various genome projects continue to produce results, this assumption becomes increasingly true, but is likely to remain somewhat problematic for the foreseeable future. This problem is due to a combination of the wide range of potential organisms used as model systems coupled with both an inability to accurately predict the proteins produced by a genome and a small but real error rate in the experimentally determined genomic sequences. An additional possible weakness of protein identification schemes involving data base searching lies in the realm of unanticipated or unknown post-translational modifications. Characterizing proteins that fall into such gaps of the data base searching routines is the subject of the present work.

Sequences of unknown peptides may be determined by chemical and enzymatic means [10] without resorting to mass spectrometry. In general however, these 
approaches are hampered both by post-translational modifications of amino acids, particularly at the terminal residues, and by the need for relatively large amounts of material. On the other hand, de novo sequencing by mass spectrometry is not yet widely used, most likely because of the difficulties associated with interpretation of high and low energy CID spectra. Additionally, successful de novo sequencing requires full sequence coverage, thus demanding better quality spectra than those typically used for data base searching. Beginning with electron ionization spectra of peptides [11], and applied to FAB spectra [12], a method of developing small peptide sub-sequences that could be extended to progressively larger fragments was developed and later applied to both high and low energy CID spectra $[13,14]$.

A very different alternative approach is presented in a recent paper [15]. The authors employ a novel extension of tandem mass spectrometry, available only for tandem-in-time trapping instruments, in which correlations between first and second order fragmentation spectra $\left(\mathrm{MS}^{3} / \mathrm{MS}^{2}\right)$ are used to determine sequences de novo.

To date, however, the most fruitful approaches to de novo sequencing have employed the principles of graph theory to both low [16-19] and high energy CID spectra [20]. The advantage to these methods is that sequences are built from the data directly, and rely on the spectra themselves rather than on predictions of sequence. With these theoretical schemes, it is possible to develop results directly from the observed data. We find that the MS/MS spectra produced by the TOF-TOF are of such a quality that sequence determinations based strictly on these spectra are both exact and straightforward to obtain. While the software used in the present work has not advanced to the level of that used in previous applications nor does it take advantage of the mass accuracy provided by the spectrometer, the success resulting from its application promises that further development may be very worthwhile.

Proteins used in this work were part of ongoing studies of the biochemical mechanism of membrane fusion being conducted in the Laboratory of Cellular and Molecular Biophysics (LCMB), National Institute of Child Health and Human Development (NICHD). For reasons of accessible biochemistry, the model system chosen for these studies are the secretory "cortical" vesicles $(\mathrm{CV})$ of sea urchin, S. purpuratus, eggs. Extensive biochemical work [21-23] in LCMB over the last several years has shown that the protein, or set of proteins, responsible for the actual membrane fusion process is not a member of any of the family of protein complexes that have previously been hypothesized to carry out the fusion role. This fact, coupled with no better than a "sparse" representation of sea urchin proteins in current data bases, suggests that the protein of interest is likely to be unknown. It is probable that this fusogenic protein, when found, will be homologous to similar proteins in higher organisms, but those proteins are also unknown or unidentified at present. Thus, for the biochemical problem of interest, there is a real need for the ability to determine a sufficient level of sequence information to establish where further biochemical and molecular biological work should be focused. Our goal in this work was to take isolated hydrophobic CV proteins and obtain as much sequence information as possible from them, focusing on selecting previously undescribed proteins as candidates for future work.

\section{Methods}

\section{Sample Preparation}

In-gel digestion of the SDS/PAGE separated proteins was carried out using a procedure similar to that published by the Association of Biomolecular Resource Facilities (ABRF) [24]. The protein bands were excised, washed, and in-gel digested using modified porcine trypsin (Promega, Madison, WI). The resulting peptides were extracted in two steps, partially dried, redissolved in $0.1 \%$ TFA (Aldrich, Milwaukee, WI); salts were removed using C18-ZipTip ${ }^{\circledR}$ (Millipore, Bedford, MA) solid phase extractions into $10 \mathrm{uL}$ of $1: 1 \mathrm{0.1 \%}$ TFA: acetonitrile.

\section{Non-TOF-TOF Analyses}

Protein digests were evaluated for suitability, in terms of digestion effectiveness, for TOF-TOF investigation using a MALDI-TOF instrument. A Voyager DE-STR (Applied Biosystems, Framingham, MA) operated in the reflector mode was used for these analyses. Samples for both peptide mass fingerprinting and TOF-TOF analysis were prepared using about $10 \%$ of the extracted digest, such that $1 \mathrm{uL}$ of the extracted digest was applied to a MALDI sample plate along with an equal volume of matrix solution (saturated solution of acyanocinnamic acid [Aldrich] in 1:1-0.1\% TFA:acetonitrile).

In addition to the TOF-TOF analyses, an LC-MS/MS analysis was performed on a portion of the extracted peptides. The extracts were partially dried and resuspended in $9 \mathrm{uL}$ of $0.1 \%$ TFA. An aliquot of this resuspended sample, 2-5 uL, was injected onto a packed capillary gradient MAGIC LC system (Michrom Bioresources, Auburn, CA) operated at $400 \mathrm{~nL} / \mathrm{min}$ using a Michrom Magic constant pressure splitter. Samples were separated using a 10 min linear gradient, $2-85 \%$ B (A, 5\% acetonitrile in water with $0.5 \%$ acetic acid, $0.005 \%$ TFA; B, $80 \%$ acetonitrile, $10 \%$ n-propanol, $10 \%$ water with $0.5 \%$ acetic acid, $0.005 \%$ TFA) with a Vydac C18, $5 \mu \mathrm{m}$ particle, $300 \AA$ pore packing material. Columns, approximately $5 \mathrm{~cm}$ in length, were packed by hand into a $75 \mu \mathrm{m}$ i.d. fused silica capillary PicoFrit (New Objective, Woburn, MA). The LC effluent was electrosprayed directly into the sampling orifice of an LCQ DECA (Thermo Finnigan, San Jose, CA) using an 


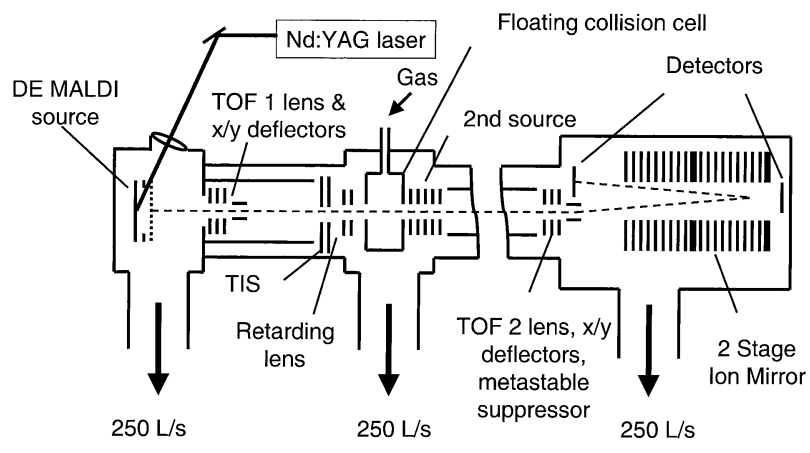

Figure 1. Schematic of MALDI TOF-TOF instrument.

adaptation of the microscale electrospray interface developed by T. D. Lee and co-workers [25]. The LCQ DECA was operated in a mode that automatically generated MS/MS spectra of the four most intense peaks present in any single scan of the ion trap, provided these peaks exceeded a preset threshold. Peptide partial internal sequence data produced by the LCMS/MS experiments were analyzed using MASCOT [5] programs.

\section{TOF-TOF Instrument}

The latest configuration of the MALDI TOF-TOF is shown in Figure 1. The concepts behind the design of the instrument, as well as more detailed descriptions of earlier versions of the instrument are found elsewhere [26]. The apparatus is similar in geometry to a conventional high quality reflectron MALDI TOF [27]. To operate the instrument in both modes, however, there are some key differences in the ion optics between the TOF-TOF and the earlier MALDI TOF reflectron instrument. In both MS and MS/MS mode, the TOF-TOF operates at $200 \mathrm{~Hz}$. In order to attain the higher repetition rate, the high voltage source 1 pulser circuit was modified, and the conventional nitrogen laser was replaced with a diode pumped Nd:YAG laser. A further substantial difference between the conventional reflectron TOF and the TOF-TOF is the use of a two stage mirror that permits better focusing across the broad energy range of fragments in MS/MS mode, without sacrificing performance in MS mode.

The MS/MS capabilities of the instrument are facilitated through the addition of the optical elements highlighted in the second source region of Figure 1-the timed ion selectors, deceleration lens, collision cell, and second source. When the instrument is operated in MS/MS mode, the first source is operated as two stage, delayed extraction, linear TOF MS, (typical acceleration voltage, $8 \mathrm{kV}$ ) with an extraction delay time that produces optimal focusing for the chosen precursor in the center of the timed ion selectors. The timed ion selectors are operated as a double sided deflection gate, with the first gate acting as the low mass gate and the second gate as the high mass gate. When the laser fires, the low mass gate is "on," and the high mass gate is "off"-all ions which enter the region of the timed ion selectors are deflected from the optical axis of the instrument. Using the precursor ion mass, the instrument geometry, and the operating voltages, the arrival time of the precursor ion to each of the gates is calculated. When the precursor enters the plane of the low mass gate, the voltage applied to the deflecting electrodes is rapidly switched off, the precursor trajectory is unaffected. After the precursor has passed through both electrodes, the high mass gate is rapidly switched on, the remaining ions are deflected from the axis of the spectrometer. The width of the precursor mass window typically functions to include the desired precursor mass and its isotope cluster. While the paired timed ion selectors are able to function with a resolution of 500 at $\mathrm{m} / \mathrm{z} 1000$, losses in sensitivity occur under those circumstances.

Collision energy in the TOF-TOF is defined by the potential difference between the source acceleration voltage and the floating collision cell, typically set to 1 $\mathrm{kV}$. The transmission of precursor ions into the collision cell is optimized by the use of a decelerating lens. There is a field free region at the potential of the collision cell, which extends from the back of the decelerating lens to the entrance of the second source. All fragments formed from the ion of interest in this region, either through unimolecular decomposition of "hot" ions (i.e., gas off spectra) or through collision induced dissociation (i.e., gas on spectra), travel with essentially the same velocity as the precursor, and thus enter the second source at the same time as the precursor ion. When the collection of precursor and fragment ions have entered the second source, a high voltage pulse is applied to the source and the ions are accelerated towards the detector. The firing of the source serves as the starting point, i.e., $t=0$, for the recording the fragment mass spectrum.

\section{Data Analysis}

Fragment ion spectra are smoothed and corrected to zero baseline using routines embedded in Data Explorer (Applied Biosystems, Framingham, MA). A list of intensity versus $\mathrm{m} / \mathrm{z}$ for that portion of the spectrum from $50 \mathrm{Da}$ to the $\mathrm{m} / \mathrm{z}$ of the precursor ion is sent to an external file as ASCII data using another embedded routine. This text file is manipulated further using a series of programs written in PERL for the purposes of this work.

At this stage of development these programs are essentially computational aids to the step-wise deduction of peptide sequences. As shown below, the fragmentation spectra are quite clean and exhibit a high signal-to-noise ratio, both of which make this rather straightforward approach to sequence determination effective. The second component of our present approach employs the relationship between the principal C-terminal, y series, and $\mathrm{N}$-terminal, b series, ions along with the mass of the precursor ion: 


$$
b_{m}=(M H+1)-y_{n}
$$

Presently, the programming does not incorporate considerations of ammonia and water losses, the "a" series ions nor any of the ion series typically associated with high energy fragmentations, $\mathrm{x}_{-}, \mathrm{w}-\mathrm{c}_{-}$, or $\mathrm{d}-$. The program uses nominal masses.

In the first step of the analysis, the text file generated in Data Explorer is read by a PERL routine and the mass of the precursor ion is inserted at the top of the list. This modified list is used in further work. A starting point for sequencing is selected, though likely candidates for $a b_{1}$ or $a y_{1}$ ion are normally selected. If the peptides originate from a trypsin digestion, the prominent $\mathrm{m} / \mathrm{z}$ 175 ion, corresponding to a C-terminal arginine, is used although complete sequences have be obtained from Aspn digestions. After reading the PERL modified mass list, another program then generates a list of candidate peaks in the spectrum corresponding to the extension of the starting mass by one residue. Candidates are reported on the basis of their observed intensities. In addition the report lists the masses and intensities of the ions that are complementary to each possible extension, see $\mathrm{Eq} \mathrm{1}$. In this fashion, one proceeds along the sequence step-wise, often from both ends of the peptide sequence. Of course, one could choose to begin at the precursor ion and extend to shorter peptides, with the complimentary ions now becoming progressively larger. Either process is continued until the peptide terminal opposite the chosen start is reached, a possibly correct sequence attained, or a point is reached beyond which it is impossible to extend the chain, i.e., a failed attempt.

The group of potential sequences are evaluated using an existing routine in the Data Explorer package. In this routine, the Ion Fragmentation Calculator, a sequence is entered and ions of the original spectrum are labeled, including sequence ions, internal fragments and losses of water and ammonia. At this time there is no scoring of the several candidate sequences, but simply a user evaluation of the "best fit". This "best fit" is based on a visual evaluation of the data-including the portion of the peaks that are labeled, i.e., can all of the peaks be explained when all possible peptide fragments are considered and stringent mass accuracy criteria are used, and whether or not the observed immonium and internal fragment ions are consistent with the proposed sequence.

An additional confirmation, in cases where peptides studied arise from proteins described in data bases, can be obtained using a widely available search algorithm, MASCOT [28]. For such confirmations, the modified text files generated with the PERL routine described above can be used to provide a file formatted appropriately for MASCOT processing. A schematic of this entire process is shown in Figure 2.

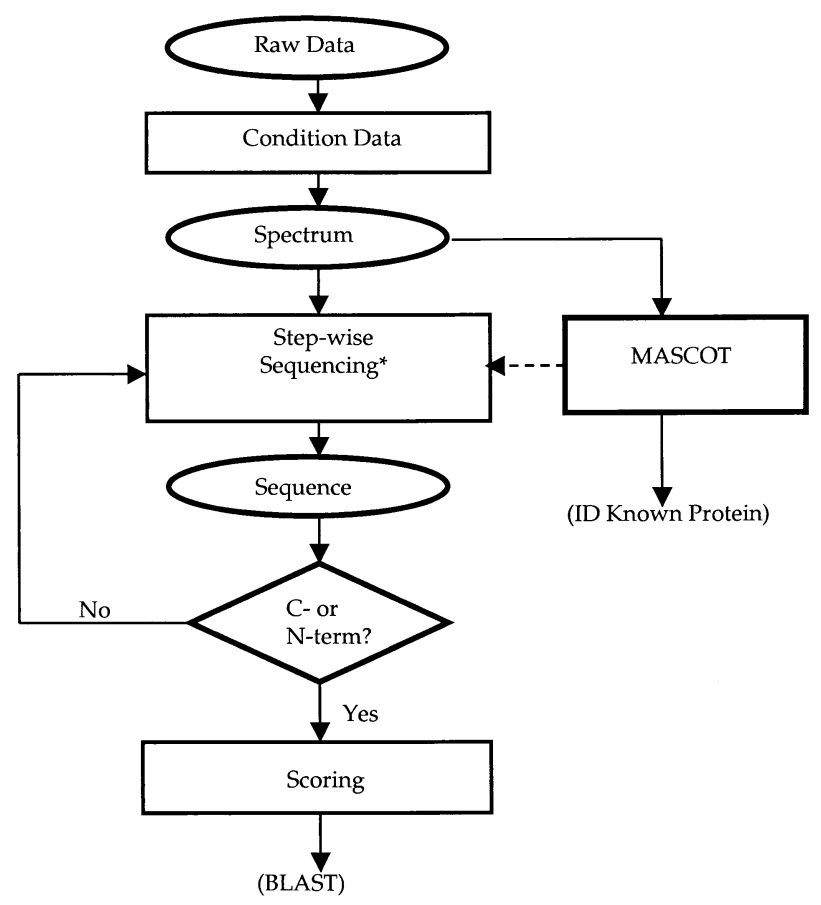

Figure 2. Schematic of de novo sequencing process. Programs were written in PERL for the processes shown in the rectangular shapes.

\section{Results and Discussion}

The first attempts at de novo sequencing of a peptide were made using the standard material angiotensin I. Fragmentation spectra were obtained from approximately 1000 laser shots of 100 fmole of this peptide in both the simple metastable decomposition mode, "gas off", and the collision induced dissociation mode, "gas on". In the latter mode, collision cell gas pressures were set at about $1 \times 10^{-6}$ torr and collision energies at about $1 \mathrm{keV}$, nominal single collision conditions. A combination of gas on and gas off spectra showed, in addition to what appeared to be a complete set of sequence ions, an almost complete set of dipeptide internal fragment ions and a number of tripeptide internal fragment ions. We realized that the combination of di- and tripeptide ions could be used by themselves, absent of virtually any other information, to develop a sequence of the precursor peptide. This fact is a consequence of a few simple combinatorial rules that can be applied to such a set of ions. We were able to show that the sequence of angiotensin I could be correctly deduced from the observed set of ions. Unfortunately, this simple and attractive approach could not be generally applied and was abandoned in future work. Subsequent efforts were directed solely to sequencing of peptides derived from sea urchin proteins.

The high signal to noise and overall appearance of a typical TOF-TOF peptide fragmentation spectrum in the gas off mode is shown in Figure 3. This spectrum was obtained from $10 \%$ of the peptide mixture extracted from an in-gel digest of a sea urchin protein, designated 

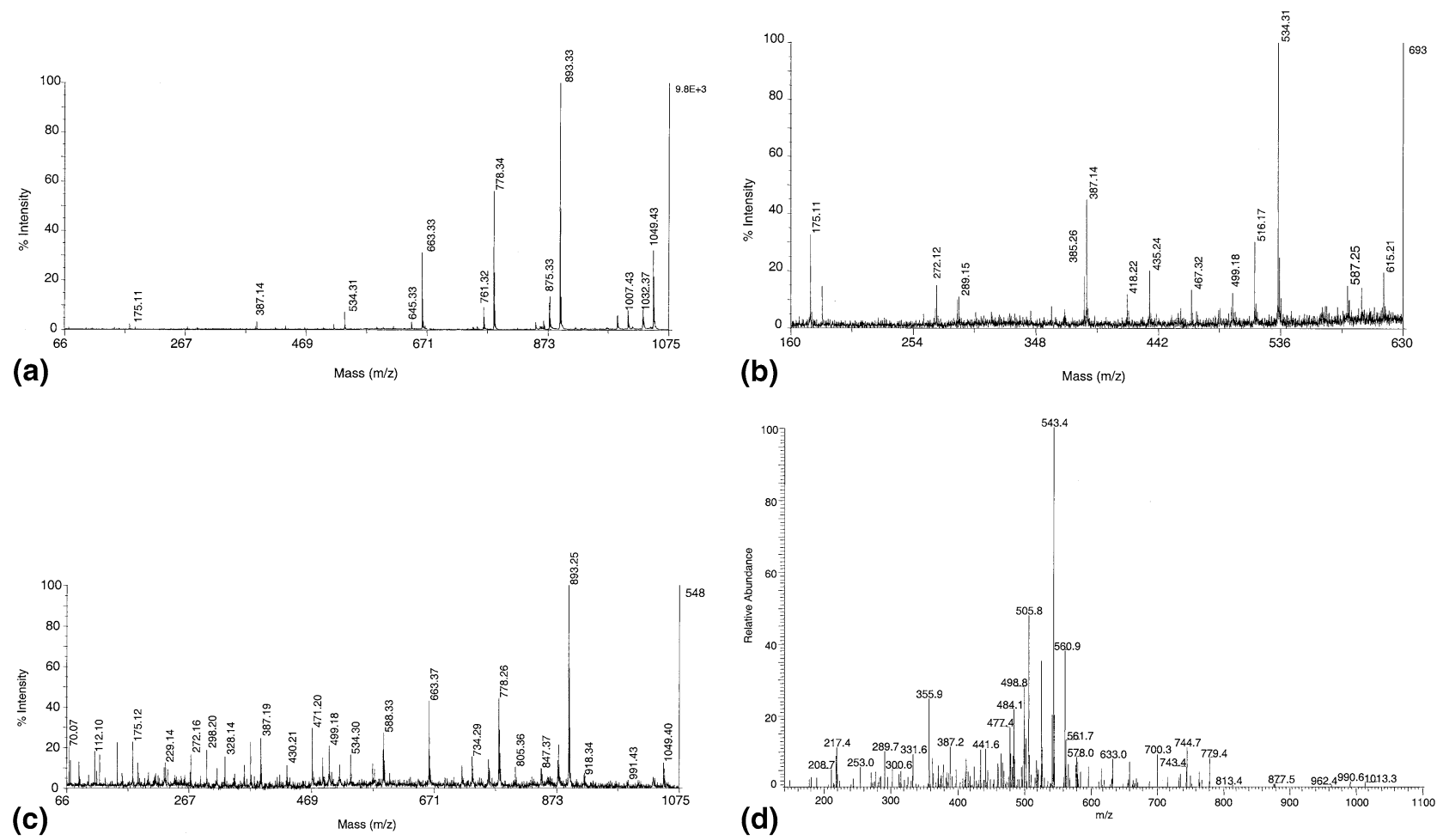

Figure 3. Comparison of TOF-TOF and LCQ DECA spectra for a precursor ion $\mathrm{m} / \mathrm{z} 1049 \mathrm{~m} / \mathrm{m} / \mathrm{z} 550$ doubly charged). (a) Full TOF-TOF gas off spectrum. (b) Low mass region of (a). (c) Full TOF-TOF gas on spectrum. (d) Ion trap MS/MS spectrum.

M7. Based on the intensity of the Coomassie Brilliant Blue band that was digested, we estimate a few pmole in the band, and thus a maximum of a few hundred fmole in the sample spotted for TOF-TOF analysis. Figure 3a shows the full mass range spectrum of a peptide of $\mathrm{MH}^{+}=1049.43$ in gas off mode, i.e., the metastable fragmentation spectrum. Note that the base peak intensity, $m / z$ 899.33, is about $10^{4}$. Figure $3 b$ is an expansion of the low mass region of the spectrum and is normalized to $\mathrm{m} / \mathrm{z} 534.31$, a low intensity ion seen in Figure 3a. The normalized intensity of Figure $3 \mathrm{~b}$ is about 700 , or approximately $7 \%$ of the base peak intensity. Inspection of $3 b$ shows a $\mathrm{S} / \mathrm{N}$ of greater than $50: 1$, and thus a $\mathrm{S} / \mathrm{N}$ for the overall spectrum approaching $10^{4}$. The gas on fragment ion spectrum for this precursor ion is shown in Figure 3c. In this case the fragmentation spectrum is clearly richer than in the gas off case, but this is a consequence of individual peptide chemistry, as seen below. The spectra shown in Figure $3 \mathrm{a}$ and $3 \mathrm{c}$ are both the result of averaging 10,000 laser shots. While the $\mathrm{S} / \mathrm{N}$ of Figure $3 \mathrm{c}$ is lower than that of Figure $3 a$, the gas on spectrum was acquired following the acquisition of nine additional 10,000 shot spectra. That is, Figure $3 \mathrm{c}$ is a 10,000 shot spectrum obtained after 100,000 prior shots from the same sample. Figure $3 \mathrm{~d}$ shows the fragmentation spectrum from the comparable doubly charged ion, $m / z 525$, obtained from the LC-MS/MS study done in parallel with the TOF-TOF analysis. In this method, $25 \%$ of the peptide extract was applied to the LC column.

MALDI TOF peptide mapping of the M7 digest yielded no clear identification of the protein. Results from parallel TOF-TOF and LC-MS/MS characterization of the peptides of the M7 band, analyses run in parallel, showed it to be a sea urchin protein known as Cortical Vesicle P18, (CV-P18). These results, summa-

Table 1. Peptides sequenced from CV-P18 protein

\begin{tabular}{|c|c|c|}
\hline Sequence & $m / z$ & $\mathrm{~T} / \mathrm{L}^{\mathrm{a}}$ \\
\hline $\mathrm{R}\langle\mathrm{DDEVFLR}\rangle \mathrm{L}$ & 893.4 & $\mathrm{~T}$ \\
\hline $\mathrm{R}\langle\mathrm{TIAFTVER}\rangle \mathrm{T}$ & 936.5 & $\mathrm{~T} / \mathrm{L}$ \\
\hline $\mathrm{K}\langle$ RDDEVFLR $\rangle \mathrm{L}$ & 1049.5 & $\mathrm{~T}$ \\
\hline $\mathrm{R}\langle\mathrm{VE}$ LFKEAGKD LK〉S & 1489.9 & $\mathrm{~L}$ \\
\hline $\mathrm{R}\langle$ LAP GLLDADADRPLFT GY LV YR $\rangle \mathrm{E}$ & 2549.4 & $\mathrm{~L}$ \\
\hline$\langle\text { DFZYER }\rangle^{\mathrm{b}}$ & 857.4 & $\bar{T}$ \\
\hline
\end{tabular}

${ }^{\mathrm{a}} \mathrm{T}=$ TOF-TOF, $\mathrm{L}=$ LCQ Ion Trap.

bPublished at $\mathrm{N}$-terminal is . .VG $\langle\mathrm{DFZYER}\rangle$. See text. 
Table 2. Summary peptide sequences from BM3- sea urchin proteins

\begin{tabular}{|c|c|c|c|c|}
\hline Band & $m / z$ & Sequence & Protein & Method $^{\mathrm{a}}$ \\
\hline \multirow[t]{3}{*}{ BM3-5 } & 1311.58 & $\langle$ TDDDWELVYR $\rangle$ & S. purpuratus GTP binding protein & $\mathrm{T}^{\mathrm{b}}$ \\
\hline & 1448.54 & $\langle$ RTPNDWELVYR $\rangle$ & S. purpuratus GTP binding protein & $\mathrm{M}(15)$ \\
\hline & 1575.67 & $\langle$ FIPPDFADGIQSIR $\rangle$ & S. purpuratus GTP binding protein & M (66) \\
\hline \multirow[t]{2}{*}{ ВM3-7 } & 852.38 & $\langle$ STPYNDR $\rangle$ & (Xenopus Mps1) & $\mathrm{T}^{\mathrm{b}}$ \\
\hline & 855 & $\langle$ SVFSYPR $\rangle$ & (Drosophila T-related protein) & $\mathrm{T}^{\mathrm{b}}$ \\
\hline \multirow[t]{4}{*}{ BM3-10 } & 1565.23 & $\langle$ HSVDFVPEAGSQHR $\rangle$ & S. purpuratus CV31-53 protein & M (67) \\
\hline & 1636.34 & $\langle\text { SALTLTFSTDPVVR }\rangle^{\mathrm{c}}$ & S. purpuratus CV31-53 protein & $M(31)$ \\
\hline & 1713.23 & $\langle$ SFESPTVNXGDEFDR $\rangle$ & S. purpuratus CV31-53 protein & $\mathrm{T}$ \\
\hline & 2147.44 & $\langle$ ALTLSSVPDLTFSTDPVVER $\rangle$ & S. purpuratus CV31-53 protein & $M(15)$ \\
\hline
\end{tabular}

${ }^{\mathrm{a}} \mathrm{T}=$ de novo by sequence extension, $\mathrm{M}()=$ MASCOT (Ion Score) where score $>40=$ identity.

$\mathrm{b}=$ not identified by MASCOT.

$\mathrm{c}=$ partial fragmentation, see text.

rized in Table 1, serve to illustrate several significant aspects of the ability to obtain clean fragment spectra with high signal to noise levels. This band was the first set of peptides to which our de novo approach was applied, and at the time they were undertaken, the identity of the protein was uncertain.

Partial or complete sequences were obtained for six peptides from this digest mixture, four using our de novo routine and three using either or both of the automated routines commonly applied to LC-MS/MS data, SEQUEST and MASCOT. Both approaches identified the CV-P18 very reliably, but there are differences between these two approaches that would be significant if the protein involved were truly unknown.

The four sequences deduced from the TOF-TOF data, designated in Table 1 with a " $\mathrm{T}$ " in the last column, were complete. Significantly, the peptide at $\mathrm{MH}^{+} 857.4$ yielded a sequence, DFZYER, where " $Z$ " is taken to be either lysine of glutamine. This sequence is part of the overall CV-P18 protein, but could not be found in a list of peptides produced by an in silico tryptic digestion of the published sequence. The reason for this peptide being absent from the list is that rather than a $\mathrm{K}$ or $\mathrm{R}$ residue at the N-terminal of the peptide, the published sequence reports a $\mathrm{G}$, thus eliminating the possibility of a peptide at the mass observed. The LC-MS/MS data set found a peptide at $m / z$ 857.4, but the automated data interpretation routines using trypsin as an enzyme could not associate this peptide with CV-P18 because of the data base error. However, a search of the database with no enzyme specificity should have identified this peptide.

Of the three peptides found by the automated searches of the LC-MS/MS data set, designated by an " $\mathrm{L}$ " in Table 1 , only one was complete, the peptide at $m / z$ 936.5. The spectrum for the ion at $m / z$ 1049.5, shown in Figure 3d, was uninterpretable by either SEQUEST or MASCOT. Furthermore, the interpretation of the ions of $\mathrm{MH}^{+}=1489.9$ and 2549.4 yielded only partial sequences which did not identify dipeptides at both ends of the $\mathrm{m} / \mathrm{z} 1489.9$ peptide and a tripeptide at the N-terminal as well as two other dipeptides of the $\mathrm{m} / \mathrm{z}$ 2549.4 peptide. The uncharacterized regions are shown in bold italics in Table 2 .
An additional aspect of the utility of TOF-TOF spectra in de novo sequencing is discussed in conjunction with Figure 4. Figure 4a is a full gas off mass spectrum of a peptide resulting from trypsin digestion of a $105 \mathrm{kD}$ protein, designated BM3-5, isolated from a different sea urchin $\mathrm{CV}$ preparation than that used to produce CVP18. The protonated precursor used in this example had a nominal $\mathrm{m} / \mathrm{z}$ of 1311 . The spectrum feature near $\mathrm{m} / \mathrm{z}$ 1104 is not associated with the peptide under study, a fact shown by the negative mass defect associated with the ion at $m / z 1104.08$ and with other ions nearby. This ion can be assigned to a fragment of matrix impurity. This gas off spectrum was used to generate a peptide sequence: TDDDWEXVYR, where the one letter amino acid code " $X$ " is taken to be either leucine or isoleucine. Several other candidate sequences were also generated including: TETDWEXVYR, TDDVAMEXVYR, and TDDDWEDPYR. None of these four candidate sequences included the abundant ion at $\mathrm{m} / \mathrm{z} 510.23$ as a sequence ion. Applying the spectrum matching routine found in Data Explorer to each of these potential sequences in turn, we concluded that the decapeptide TDDDWEXVYR was the correct sequence. The ion at $m / z 510.23$ is assigned as a water loss from the internal fragment for WELV. A search of the NCBI nonredundant data base using BLAST showed this peptide to be associated with a known sea urchin GTP binding protein.

We hoped to confirm the sequence of the GTP binding protein with the use of the more extensive fragmentation, including the production of immonium ions, that is characteristic of high energy CID and thus observed in a gas on spectrum. The full mass range gas on spectrum is shown in Figure $4 \mathrm{~b}$, again at a reduced $\mathrm{S} / \mathrm{N}$ compared to Figure 4a because of sample depletion. Note that for this sample, the gas off fragmentation spectrum is richer in the high mass region than is the gas on spectrum, in contrast to the case shown in Figure 3 . The low mass region of the gas on spectrum of the nominal $\mathrm{m} / \mathrm{z} 1311$ precursor ion is shown in Figure 4c. In addition to the strong immonium ions for the sequence proposed at $\mathrm{m} / \mathrm{z} 86(\mathrm{X}), 136(\mathrm{~T})$, and $159(\mathrm{~W})$, there are immonium ion peaks at $m / z 70(\mathrm{P}), 87(\mathrm{~N})$, and 120 (F). It should be noted that $m / z 70$ and 87 can also be ascribed to arginine, but at lower intensities than 


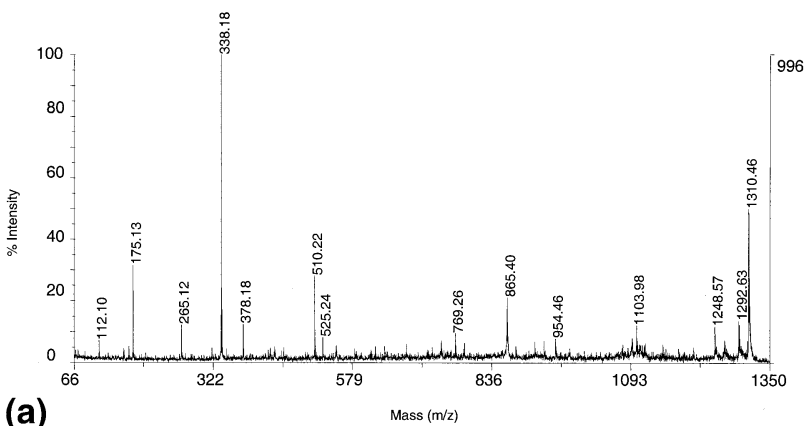

(a)

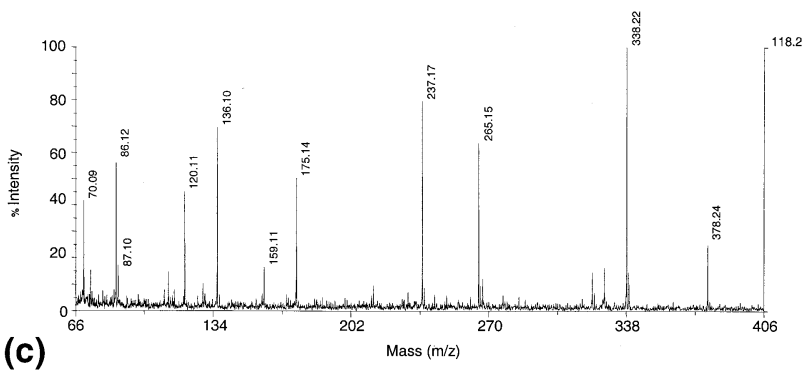

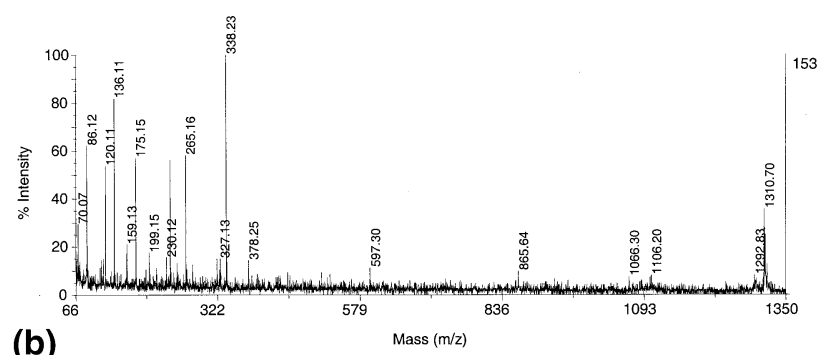

(b)

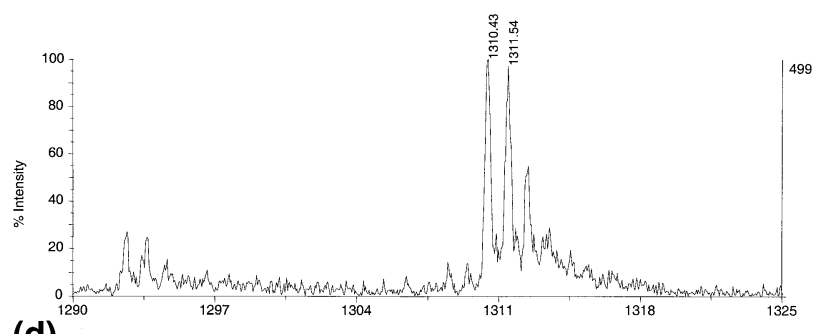

(d)

Figure 4. TOF-TOF spectrum of precursor $m / z$ 1311. (a) Gas off spectrum. (b) Gas on spectrum. (c) Low mass region of $(\mathbf{b})$. (d) Gas off precursor parent iosotope cluster.

present in this spectrum. None of the latter three residues are in the proposed sequence, and only the proline is present in the three alternates that were subsequently rejected. This apparent anomaly was resolved by close inspection of the parent ion cluster of the precursor ion, shown in Figure $4 \mathrm{~d}$. It is apparent from this isotope cluster that what was taken as a single precursor is in fact two, one at $m / z 1311.59$, sequenced from the gas off spectrum, and a second peptide with a precursor at $m / z$ 1310.70. There was sufficient information between the two spectra to obtain a sequence of this second peptide as well. We determined its sequence and, when searched against the NCBI nonredundant data base using BLAST, was shown to have extensive homology with a peptide from $\mathrm{C}$. botolinum neurotoxin. This finding may be of some interest biochemically and is being investigated further.

A total of two additional bands from the preparation of sea urchin $\mathrm{CV}$ were investigated using MALDI TOF-TOF. The results of both our de novo sequencing and the MASCOT searches of the fragmentation spectra are summarized in Table 2 . De novo sequencing identified an urchin GTP binding protein and suggested the possibility of a heretofore undescribed urchin protein in the $105 \mathrm{kDa}$ band (BM3-5). The MASCOT search of the data from this digest confirmed the presence of the GTP binding protein in matching sequences of two other peptides, one with a high enough score to confidently identify the protein on its own accord. Interestingly, MASCOT was unable to match the spectrum from the nominal 1311 precursor with the GTP binding protein, most likely because of the complexity of having a mixture present. The LC-MS/MS study of this same digest found three additional peptides associated with the urchin GTP binding protein.
De novo sequencing of the weakly stained $62 \mathrm{kD}$ band (BM3-7) gave results from two peptides as shown in Table 2, neither of which showed any homology with known sea urchin proteins using BLAST. Neither the MASCOT search of the TOF-TOF spectra nor the results from the LC-MS/MS study of the digest from this band yielded indication of the presence of known sea urchin proteins. This band is clearly a candidate for future study.

The fifteen residue peptide SFESPTVNXGDEFDR, matching a sequence from a sea urchin protein known as CV31-53, was found by de novo sequencing of the peptide ion at $m / z 1713.23$. This peptide was also found in the MASCOT search as were three other peptides from the same protein and a trypsin autolysis fragment. The two low scoring peptides found in the MASCOT search, $m / z 1636.34$ and 2147.44 were matched using spectra that were completely useless for our de novo approach because of an almost complete lack of peptide fragment ions. While these sparse spectra did not give high scores in MASCOT, the interpretations made were internally consistent.

\section{Conclusion}

We have shown that spectra resulting from TOF-TOF fragmentations, both gas off and gas on, are responsive to a straightforward approach to de novo sequencing. When applied to spectra of peptides that quite possibly originate from unknown proteins, our methods yield results that are useful in interpreting the origins of the bands and guiding future research directions. We have found that using existing automated search routines using the TOF-TOF spectra can be used as an initial screening technique for selecting peptide spectra for 
further detailed study. These notable exceptions to the utility of the more conventional methods show both the power of the present approach and the need for using de novo methods more frequently than may be otherwise appreciated. The rather elementary programs developed for this work have proven to be effective for interpreting the fragmentation spectra simply because the spectra are generally very clean and distinct. Obvious future development will focus on incorporating the concepts of the ion family or sequence spectrum [1620 ], as well as some scoring of the several candidate sequences.

\section{Acknowledgments}

The authors thank Jonathan Epstein, OSD, NICHD, and Chadwick Sessions, Department of Computer Science, University of Maryland, Baltimore County for the immense help and support they gave one of them (ALY) in using PERL. The authors also thank Karl Clauser, Millenium Pharmaceuticals for his generous and helpful discussions of data base searching and de novo algorithms.

\section{References}

1. Mann, M.; Hoejrup, P.; Roepstorff, P. Use of Mass Spectrometric Molecular Weight Information to Identify Proteins in Sequence Databases. Biol. Mass Spectrom. 1993, 22(6), 338-345.

2. Clauser, K.; Hall, S.C.; Smith, D.M.; Webb, J.W.; Andrews, L.; Tran, H.M.; Epstein, L.B.; Burlingame, A.L. Rapid Mass Spectrometric Peptide Sequencing and Mass Matching for Characterization of Human Melanoma Proteins Isolated by TwoDimensional PAGE. Proc. Natl. Acad. Sci. U.S.A. 1995, 92, 5072-5076.

3. Zhang, W.; Chait, B. ProFound: An Expert System for Protein Identification Using Mass Spectrometric Peptide Mapping Information. Anal. Chem. 2000, 72, 2482-2489.

4. Eriksson, J.; Chait, B.; Fenyo, D. A Statistical Approach for Testing the Significance of Mass Spectrometric Protein Identification Results. Anal. Chem. 2000, 72, 999-1005.

5. Perkins, D.; Pappin, D.J.C.; Creasy, D.M.; Cottrell, J.S. Probability-Based Protein Identification by Searching Sequence Databases Using Mass Spectrometry Data. Electrophoresis 1999, 20, 3551-3567.

6. Clauser, K.; Baker, P.; Burlingame, A. Role of Accurate Mass Measurements $(+/-10 \mathrm{ppm})$ in Protein Identification Strategies Employing MS and MS/MS. Anal. Chem. 1999, 71, 2871.

7. Egelhofer, V.; Bussow, K.; Luebbert, C.; Lehrach, H.; Nodhof, E. Improvements in Protein Identification by MALDI-TOF-MS Peptide Mapping. Anal. Chem. 2000, 72, 2741-2750.

8. Eng, J. K.; McCormack, A. L.; Yates, J. R., III. An Approach to Correlate Tandem Mass Spectral Data of Peptides with Amino Acid Sequences in a Protein Database. J. Am. Soc. Mass Spectrom. 1994, 5, 976-989.

9. Mann, M.; Wilm, M. Error Tolerant Identification of Peptides in Sequence Databases by Peptide Sequence Tags. Anal. Chem. 1994, 66, 4390-4399.

10. Tsugita, A.; van der Broek, R.; Przybylski, M. Exopeptidase Digestion in Combination with Field Desorption Mass Spec- trometry for Amino Acid Sequence Determination. FEBS Lett. 1982, 137, 19-24.

11. Bieman, K.; Cone, C.; Webster, B.R.; Arsenault, G.P. Determination of the Amino Acid Sequence in Oligopeptides by Computer Interpretation of High Resolution Mass Spectra. J. Am. Chem. Soc. 1966, 88, 5598-5606.

12. Siegel, M.; Bauman, N. An Efficient Algorithm for Sequencing Peptides Using Fast Atom Bombardment Mass Spectra. Biomed. Environ. Mass Spectrom. 1988, 15, 333-343.

13. Johnson, R.; Bieman, K. Computer Program (SEQPEP) to Aid in the Interpretation of High-Energy Collision Tandem Mass Spectra of Peptides. Biomed. Environ. Mass Spectrom. 1989, 18, 945-957.

14. Yates, J.; Griffin, P.; Hood, L. In Techniques in Protein Chemistry, Vol. II; Villafranca, J, Ed.; Academic Press: San Diego, 1991; pp 477-489.

15. Zhang, Z.; McElvain, J. De Novo Peptide Sequencing by Two-Dimensional Fragment Correlation Analysis. Anal. Chem. 2000, 72, 2337-2350.

16. Taylor, J.; Johnson, R. Sequence Database Searches via de Novo Peptide Sequencing by Tandem Mass Spectrometry. Rapid Commun. Mass Spectrom. 1997, 11, 1067-1075.

17. Dancik, V.; Addona, T.A.; Clauser, K.R.; Vath, J.E.; Pevzner, P.A. De Novo Peptide Sequencing via Tandem Mass Spectrometry. J. Comp. Biol. 1999, 6, 327-342.

18. Taylor, J.; Johnson, R. Implementation and Uses of Automated de Novo Peptide Sequencing by Tandem Mass Spectrometry. Anal. Chem. 2001, 73, 2594-2604.

19. Chen, T.; Kao, M.-Y.; Tepel, M.; Rush, J.; Church, G.M. A Dynamic Programming Approach to De Novo Peptide Sequencing via Tandem Mass Spectrometry. J. Comput. Biol. 2001, 8, 325-337.

20. Hines, W.M.; Falick, A.M.; Burlingame, A.L.; Gibson, B.W. Pattern-Based Algorithm for Peptide Sequencing from Tandem High Energy Collision-Induced Dissociation Mass Spectra. J. Am. Soc. Mass Spectrom. 1992, 3, 326-336.

21. Zimmerberg, J.; Blank, P.S.; Kolosova, I.; Cho, M.-S.; Tahara, M.; Coorssen, J.R. A Stage-Specific Preparation to Study the Ca2+-Triggered Fusion Steps of Exocytosis: Rationale and Perspectives. Biochemie 2000, 82, 303-314.

22. Tahara, M.; Coorssen, J.R.; Timmers, K.; Blank, P.S.; Whalley, T.; Scheller, R.; Zimmerberg, J. Calcium Can Disrupt the SNARE Protein Complex on Sea Urchin Egg Secretory Vesicles Without Irreversibly Blocking Fusion. J. Biol. Chem. 1998, 273, 33667-33673.

23. Coorssen, J. R.; Blank, P.S.; Tahara, M.; Zimmerberg, J. Biochemical and Functional Studies of Cortical Vesicle Dusion: The SNARE Complex and Ca2+ Sensitivity. J. Cell Biol. 1998, 143, 1845-1847.

24. Facilities A.o.B.R., Representative "In-Gel" Digestion Protocol for Proteins in SDS PAGE Gel www.abrf.org/ABRF/ResearchCommittees/intprotseqrescomm.html 1997.

25. Davis, M.T.; Stahl, D.C.; Hefta, S.A.; Lee, T.D. A Microscale Electrospray Interface for On-Line, Capillary Liquid Chromatography/Tandem Mass Spectrometry of Complex Peptide Mixtures. Anal. Chem. 1995, 67, 4549-4556T.

26. Vestal, M.; Campbell, J. Tandem Time-of-Flight Mass Spectrometry. J. Am. Soc. Mass Spectrom. 2002, unpublished.

27. Vestal, M.; Juhasz, P.; Martin, S. Delayed Extraction Matrixassisted Laser Desorption Time-of-Flight Mass Spectrometry. Rapid Commun. Mass Spectrom. 1995, 9, 1044-1050.

28. MASCOT http://www.matrixscience.com 2001. 